\title{
Acknowledgement to Reviewers of Micromachines in 2018
}

Micromachines Editorial Office

MDPI, St. Alban-Anlage 66, 4052 Basel, Switzerland

Published: 9 January 2019

Rigorous peer-review is the corner-stone of high-quality academic publishing. The editorial team greatly appreciates the reviewers who contributed their knowledge and expertise to the journal's editorial process over the past 12 months. In 2018, a total of 673 papers were published in the journal, with a median time to first decision of 15 days and a median time to publication of 38 days. The editors would like to express their sincere gratitude to the following reviewers for their cooperation and dedication in 2018:

Aaen, Peter H.

Aas, Mehdi

Abad, Manuel David

Abate, Yohannes

Abdollahzadeh Jamalabadi, M. Y.

Abdul Samad, Yarjan

Abdulrahim, Mujahid

Abelmann, Leon

Abhyankar, Vinay

Abidi, Khalid

Abolfazl, Zahedi

Abolhasani, Milad

Abou-Saleh, Radwa H.

Abramovich, Haim

Adamu-Lema, Fikru

Aghababaei, Ramin

Aguilella-Arzo, Marcel

Ahmed, Riaz

Ahmmed, K.M. Tanvir

Ahsan, Md. Shamim

$\mathrm{Ai}, \mathrm{Ye}$

Akafuah, Nelson K.

Akram, Muhammad Nadeem

Al-Ameri, Talib

Alessio, Verna

Alfano, Brigida

Algorri, José Francisco

Ali, Dr. Md. Azahar

Alirezaee, Shahpour

Alsaleem, Fadi

Álvarez, Mar

Amarie, Dragos

Amit, Iddo
An, Sangmin

An, Seongpil

Anders, Jens

Angotzi, Gian Nicola

Ansari, Azadeh

Ansari, Farhan

Anthony, Carl

Aral, Mustafa

Aramesh, Morteza

Arefin, Md. Shamsul

Ariga, Katsuhiko

Arnold, Walter

Arscott, Steve

Aryasomayajula, Aditya

Asadnia, Mohsen

Asokanthan, Samuel F.

Assaf, Tareq

Attar, Hooyar

$\mathrm{Au}, \mathrm{Sam} \mathrm{H}$.

Audette, Michel

Avci, Ebubekir

Aw, Kean

Azar, Massood

Azimi, Soheil

Azizi, Ebrahim

Bae, Hyungdae

Baek, Donghyun

Bagdziunas, Gintautas

Bagolini, Alvise

Bai, Jing

Balasingam, Suresh Kannan

Banerjee, Ashis Gopal

Bannock, James H. 
Bao, Yinyin

Barbato, Felicia Carla Tiziana

Barbato, Marco

Barbe, Jean-charles

Barberi, Riccardo

Bardweel, Hamzeh

Barfidokht, Abbas

Barletta, Diego

Baron, Thomas

Barralon, Pierre

Barreto, Armando

Barshtein, Gregory

Bartlett, Michael D.

Bartsch, Heike

Basha, Mohamed

Basit, Munshi Mahbubul

Basu, Anirban

Batra, A. K.

Baù, Marco

Bauer, Ralf

Beheim, Glenn M.

Beilliard, Yann

Beker, Levent

Beleggia, Marco

Belfiore, Nicola Pio

Bella, Federico

Bellantone, Vincenzo

Belo, Joao Horta

Ben, Yueyang

Benedic, Fabien

Benseman, Timothy M.

Berg-Sorensen, Kirstine

Bernard, Yves

Bernini, Romeo

Bertocci, Francesco

Bertsch, Arnaud

Besagni, Giorgio

Besozzi, Edoardo

Bhadra, Sharmistha

Bhakhri, Vineet

Bhalla, Nikhil

Bhaskaran, Madhu

Bhattacharjya, Dhrubajyoti

Bhattacharya, Sriparna

Bianucci, Pablo

Bieniek, Tomasz

Bittner, Achim

Bjorninen, Toni

Black, Bryan James

Blanche, Pierre-alexandre

Bleichner, Martin

Bobinger, Marco
Boek, Edo

Bogdanowicz, Robert

Boland, Jessica L.

Borges Sebastião, Israel

Bort, Juan M. Andrés

Bose, Saurabh

Bota, Sebastia

Boudaden, Jamila

Boudot, Rodolphe

Bourouina, Tarik

Boussommier-Calleja, Alexandra

Boutami, Salim

Boyd, Anthony

Bragheri, Francesca

Bramesfeld, Goetz

Bratsun, Dmitry

Brevik, Iver

Brisk, Philip

Brouzes, Eric

Brunet, Philippe

Brunton, Emma

Bucci, Davide

Buj, Irene

Burugupally, Sindhu Preetham

Cabot, Joan Marc

Cajal, Carlos

Cakmakyapan, Semih

Calabrese, Luigi

Calaza, Carlos

Caliano, Giosue

Calvo, Roque

Camarchia, Vittorio

Camarillo, David

Camboni, Domenico

Caminero, M.A.

Campos, Manuel

Canfield, Brian

Cano, Juan Luis

Canpolat, Cetin

Cao, Huayu

Caporali, Stefano

Cardenas, Jaime

Carey, Benjamin

Carou, Diego

Carugo, Dario

Casas Bedoya, Alvaro

Caselli, Federica

Cassidy, John F.

Castellanos-Ramos, Julián

Cataldi, Pietro

Catarino, Susana

Cavicchi, Kevin 
Cazzanelli, Massimo

Cegla, Frederic

Cellini, Filippo

Ceylan, Hakan

Chaisakul, Papichaya

Chakraborty, Monojit

Chalmers, Jeffrey J.

Chan, King Yuk

Chan, Pak

Chan, Yung-Kuan

Chandrahalim, Hengky

Chang, Bo

Chang, Chih-Wei

Chang, Lingqian

Chang, Wenlong

Chang, Yoon-Seok

Channon, Robert

Charkhkar, Hamid

Charlton, Peter

Charmet, Jerome

Chaspari, Theodora

Chaudhary, Varun

Chavoshi, Saeed Zare

Chen, Chia-Hung

Chen, Chia-Yuan

Chen, Chi-Feng

Chen, Chihchen

Chen, Chunhui

Chen, Daiqin

Chen, Heping

Chen, Jinju

Chen, Jun

Chen, Mohan

Chen, Nanguang

Chen, Pengyu

Chen, Qiushu

Chen, Ruimin

Chen, Shao-Wen

Chen, Tao-Hsing

Chen, Xing

Chen, Yuanliu

Chen, Yunhan

Chen, Zhenghua

Cheng, Cheng

Cheng, Chiang-ho

Cheng, Guangming

Cheng, Zengguang

Cheng, Zhenzhou

Chesneau, Xavier

Chiang, Cheng-Ta

Chiang, Chia-Chin

Chien, Jun-Chau
Chin, Jit Kai

Chinappi, Mauro

Chiolerio, Alessandro

Cho, Chungyeon

Cho, Hansang

Cho, Young-Rae

Choi, Heekyu

Choi, Hyo-Jick

Choi, Jungil

Choi, Jungwook

Choi, Kyung Hyun

Choi, Seung-Bok

Choi, Sungyoung

Choi, WooSeok

Chow, Hwang-Cherng

Christoffer, Abrahamsson

Chua, Beelee

Chuang, Cheng-Hsin

Chung, Ying-Chien

Churchill, Hugh

Cimalla, Volker

Cinquemani, Simone

Clement, Yuen

Climente-Alarcon, Vicente

Cogan, Stuart

Cohen, Estelle

Coimbatore Balram, Krishna

Collin, Eddy

Collins, David J.

Comstock, Matthew

Coninck, Joel De

Connelly, Daniel

Constantoudis, Vassilios

Cooke, Michael

Copic, Davor

Coppola, Sara

Cordill, Megal

Cortese, Barbara

Coskun, Bulut

COURTIAL, Edwin-Jofrey

Coy, Emerson

Cozzoli, P. Davide

Crane, Nathan

Croce, Anna Cleta

Croissant, Jonas

Croizet, Cédric

Croquette, Vincent

Crupi, Giovanni

Cui, Bo

Culebras, Mario

Culha, Utku

D'Alonzo, Marco 
Da Silva, Jaime Pedro Oliveira

Dagdeviren, Canan

Dai, Jing

Dalla Costa, Giuseppe

D'andrea, Cristiano

D'Andreagiovanni, Fabio

Das, Pradip

Dash, Biraja

Davaji, Benyamin

Dawson, Jeremy

De Aza, Piedad N.

De Boer, Greg

De Dood, Michiel J.

De Gasperis, Giovanni

De La Fuente, Germán Francisco

De Marco, Carmela

De Munari, Ilaria

De Nijs, Bart

Dean, Robert

Defay, Emmanuel

Degennaro, Leonardo

Dehaeck, Sam

Delfs, Jens-Olaf

Delivopoulos, Evangelos

Dellar, Paul J.

Dell'Olio, Francesco

Delnavaz, Aidin

DeMiguel-Ramos, Mario

D'Emilia, Giulio

Demirci, Ibrahim

Deng, Nan-Nan

Deshpande, S.R.

Destgeer, Ghulam

Devasahayam, Sheila

Di Bartolomeo, Antonio

Di Benedetto, Luigi

Di Caprio, Giuseppe

Di Schino, Andrea

Di Sieno, Laura

Dianat, Pouya

Diban, Nazely

Dickert, Franz L.

Didar, Tohid

Diller, Eric

Dimaki, Maria

Ding, Junjun

Ding, Wendu

Ding, Xiaoyun (Sean)

Ding, Zhaoyang

Dinh, Toan

Dixit, Chandra K.

Do, Thanh Nho
Dohi, Tetsuji

Dombek, Grzegorz

Dong, Daoyi

Dong, Junliang

Dong, Mingdong

Drese, Klaus Stefan

Druffel, Thad

Dryden, Michael DM

Du, E (Sarah)

Duan, Xiyu

Dubuc, David

Duchamp, Martial

Ducobu, François

Duffy, Simon

D'Urso, Gianluca

Dusny, Christian

Dziedzic, Andrzej

Eberle, Patric

Ebralidze, Iraklii I.

Eddington, David

Eder, Stefan

Edirisinghe, Mohan

Eftekhar Azam, Saeed

Ehrmann, Andrea

Elbuken, Caglar

Elg, Daniel

El-Heliebi, Amin

Elisa, Kallioniemi

Ellett, Felix

Elmazria, Omar

Elsayed, Mohannad

Elshimali, Yahya I.

Emadi, Arezoo

Enguita, José María

Enteria, Napoleon A.

Eral, H. Burak

Erdmann, Andreas

Ereifej, Evon

Ertl, Peter

Esashi, Masayoshi

Escobedo, Carlos

Escobedo-Lucea, Carmen

Etcheverry, Sebastian

Etienne, Dague

Facchini, Francesco

Falcucci, Giacomo

Fallon, James

Fan, Linran

Fang, Hui

Farokhi, Hamed

Fassi, Irene

Feiertag, Gregor 
Fekete, Zoltán

Ferhan, Abdul Rahim

Fernandez Rivas, David

Fernandez-Prieto, Armando

Ferrandiz Bou, Santiago

Ferrari, Michele

Ferraro, Davide

Ferraro, Pietro

Filippeschi, Alessandro

Finkler, Amit

Finney, Eric E.

Firebaugh, Samara L.

Flowers, George

Fodor, Petru S.

Folch, Albert

Foudeh, Amir

Foulds, Ian

Fournel, Frank

Fournelle, Marc

Francés-Soriano, Laura

Frense, Dieter

Friedt, Jean Michel

Friedt, Jean-Michel

Frisch, Benjamin

Fromen, Cathy

Froriep, Ulrich P

Fukuda, Takeshi

Funari, Riccardo

Funfschillin, Denis

Fung, Wai-Keung

Fuso, Francesco

Gaczynska, Maria

Gai, Ya

Gaitas, Angelo

Galleani, Lorenzo

Ganesana, Mallikarjunarao

Ganser, Christian

Gao, Jie

Gao, Nan

Gao, Weibo

Gao, Xuan

Gao, Yuji

Garbovskiy, Yuriy

García, Nuria

García-Miquel, Hector

García-Sánchez, Pablo

Gargiulo, Gaetano

Gargiulo, Gaetano D

Gebicki, Jacek

Gębicki, Jacek

Gennari, Oriella

Gentile, Vittorio
Georgiou, Emmanuel P.

Gerber, Doron

Gerdon, Aren

Ghafar-Zadeh, Ebrahim

Ghayesh, Mergen

Giakos, George

Giani, Alain

Giannakoudakis, Dimitrios

Giannetti, Ambra

Gibbs, John

Gielen, Fabrice

Giménez-Gómez, Pablo

Gimsa, Jan

Giorgio, Ivan

Giubileo, Filippo

Gizdavic-Nikolaidis, Marija

Glowacz, Adam

Goel, Saurav

Gogneau, Noelle

Gokhale, Vikrant Jayant

Golub, Victor V.

Gómez Salinas, Dídac

Gomez, Alessandro

Gong, Hua

González, Icíar

González, María Ujué

González-Romero, Elisa

Goossen, Keith

Gopalan, Sai Anand

Gordillo, Jose Manuel

Grattieri, Matteo

Gray, Michael D.

Greene, Nathaniel

Greener, Jesse

Griffini, Gianmarco

Grigsby, Christopher L.

Grijalvo, Santiago

Grondin, Francois

Gross, Andrew James

Grossi, Marco

Grünberger, Alexander

Gspann, Thurid

Gu, Wenting

Gu, Xiaodan

Guan, Weihua

Guaraldo, Thais Tasso

Guder, Firat

Guénolé, Julien

Guijt, Rosanne M.

Guiney, Linda M.

Guix, Maria

Gumuscu, Burcu 
Gunkel, Ilja

Guo, Peijun

Guo, Qingbo

Guo, Zhanhu

Gutruf, Philipp

Guzman, Marcelo

Haddadi, Abbas

HADDAG, Badis

Haesler, Jacques

Hafiz, Md Abdullah Al

Haghshenas, Meysam

Haidar, Riad

Hakem, Nadir

Halldórsson, Skarphéðinn

Hamon, Morgan

Han, Yen-Lin

Hane, Kazuhiro

Hao, Guangbo

Hao, Nanjing

Harnois, Maxime

Hasanov, Namig

Hashim, Hairulazwan

Hasni, Hassene

Hassan, Sammer-ul

Hassanin, Hany

Hattar, Khalid

Hayakawa, Tohru

$\mathrm{He}$, Mei

$\mathrm{He}$, Yong

Heepe, Lars

Hegemann, Dirk

Heise, Bettina

Hejazian, Majid

Hellström, Per-Erik

Hemsel, Tobias

Herkommer, Alois

Hernando, Jorge

Herrera-May, Agustín

Herrero, Rebeca

Herth, Etienne

Hesketh, Peter

Hida, Hirotaka

Hidrovo, Carlos H.

Higgins, Simon

Hinchet, Ronan

Hirai, Yoshikazu

Hirtz, Michael

Hjelme, Dag Roar

Ho, Megan Yi-Ping

Hoath, Stephen

Hoettges, Kai

Hoffmann, Rolf
Homsy, Alexandra

Honda, Takashi

Hong, Sukjoon

Hoshiba, Takashi

Hossain, Shakhawat

Hossan, Mohammad Robiul

Howlader, Matiar

Hsieh, Kuangwen

Hsu, Wei-Lun

$\mathrm{Hu}$, Chunxiao

$\mathrm{Hu}$, Guoqing

$\mathrm{Hu}$, Zhongxu

Hua, Tao

Huang, Chih-Hsien

Huang, Chi-Hsien

Huang, $\mathrm{Hu}$

Huang, Kuo-Cheng

Huang, Po-Hsun

Huang, Qijin

Huang, Shengxi

Huang, Shyh-Chour

Huang, Yanbo

Huang, Ying

Huang, Yu-Hsi

Huck, Alexander

Hülsenberg, Dagmar

Hummon, Matthew

Hunstig, Matthias

Huo, Dehong

Hwang, Chi-Hung

Hwang, David J.

Hwang, Dong-Hwan

Hwang, Jae Youn

Hwang, Suk-Won

Hwang, Yongha

Hwang, Yongsung

Hwu, Jenn-Gwo

Hyun, Kyung-A

Iatsunskyi, Igor

Ignés-Mullol, Jordi

Ikmo, Park

Iliescu, Ciprian

Iliopoulos, Konstantinos

Im, Sung Gap

Ino, Kosuke

Ishida, Tadashi

Ishikawa, Takuji

Islam, Kamrul

Ito, So

Itoh, Tamitake

Iwami, Kentaro

Iwase, Eiji 
Iwata, Tatsuya

Iza, Felipe

Jackson, Mark

Jackson, Nathan

Jakieła, Sławomir

Jakubik, Wiesław P.

James, Sagil

Jang, JongMoon

Jayaram, Dhanya Thamaraparambil

Jeon, Seokwoo

Jeong, Chang Kyu

Jeong, Jae-Woong

Jeong, Ok Chan

Jeong, Seung Hee

Jeong, Yaesuk

Jia, Ru

Jia, Yu

Jian, Jie

Jiang, Kun

Jiang, Song

Jiao, Pengcheng

Jin, Xiaoliang

Jing, Qingshen

Jing, Wuming

Jo, Sae Byeok

Johnson, Stephanie

Jones, J Cliff

Jones, Matthew R

Jones, Philip

Joshi, Niravkumar

Joung, Daeha

Joung, Hyou-Arm

Jovanović, Dragana

Juárez, Jaime Javier

Jugessur, Aju

Jung, Daewoong

Jung, Hyun Jun

Jung, Hyungil

Jung, Sungjune

Juodkazis, Saulius

Jurado-Sánchez, Beatriz

Kaajakari, Ville

Kacem, Najib

Kacher, Josh

Kadasala, Naveen Reddy

Kahrs, Lueder A.

Kaiser, Susanna

Kaji, Noritada

Kalantar-Zadeh, Kourosh

Kale, Akshay

Kaltsas, Grigoris

Kamath, Rahul
Kamenetska, Masha

Kameoka, Jun

Kanda, Kensuke

Kaneko, Yoshihisa

Kanematsu, Hideyuki

Kang, Dong-Ku

Kang, Pilgyu

Kang, Seong Jun

Kang, Xin

Kang, Yang Jun

Kanik, Mehmet

Kannappan, Ramaswamy

Kano, Shinya

Kantak, Chaitanya

Kaplan, Andrey

Karakasidis, T.E.

Karanassios, Vassili

Kärnfelt, Camilla

Kashaninejad, Navid

Kaushik, Ajeet

Kavehei, Omid

Kawashima, Kenji

Kaya, Tolga

Kazem, Navid

Keating, Adrian

Keedwell, Edward

Kehl, Florian

Keleher, Jason J.

Keller, Clément

Kelley, Joshua

Keszler, Agnes

Khalid, Ata

Khaliq, Jibran

Khan, Muhammad

Khoshmanesh, Khashayar

Kiani, Amirkianoosh

Kiefer, Rudolf

Kilic, Zekai Murat

Kim, Chang Nyung

Kim, Chang-Jin

Kim, Chulhong

Kim, Chung-Soo

Kim, Daewon

Kim, Dae-Yoon

Kim, Dongin

Kim, Hee-Seok

Kim, Hong Nam

Kim, Jaekyun

Kim, Jaemin

Kim, Jaeyoun

Kim, Jehyung

Kim, Jin Sik 
Kim, Jinwook

Kim, Kwanoh

Kim, Kyunghoon

Kim, SangHyeon

Kim, Sangkil

Kim, Seokmin

Kim, Sung Jae

Kim, Sung-Jin

Kim, Yoonseob

Kirchner, Robert

Kirihara, Soshu

Kirillova, Alina

Kitamura, Akira

Kitchen, Philip

Kityk, Iwan

Knappe, Svenja

Knospe, Carl R.

Ko, Hyoungho

Ko, Seong-Young

Ko, SeungDeok

Kobayashi, Atsushi

Kobayashi, Makiko

Kobtsev, Sergey

Koh, Ahyeon

Koh, Kah How

Kokkoris, George

Koltay, Peter

Konda Gokuldoss, Prashanth

Kondoh, Jun

Kong, Tian Fook

Könözsy, László

Konstantaki, Maria

Kontziampasis, Dimitrios

Koo, Chiwan

Kootala, Sujit

Koppányi, Zoltán

Koppes, Ryan

Korivi, Naga

Korobiichuk, Igor

Korsunsky, Alexander M.

Korvink, Jan

Kotsuchibashi, Yohei

Kottapalli, Ajay Giri Prakash

Kouh, Taejoon

Koumoulos, Elias P.

Kouza, Maksim

Kovalchenko, Andrii M.

Kozai, Takashi

Kozlowska, Justyna

Kraft, Thomas

Krebs, Guillaume

Kreth, Phillip
Krolczyk, Grzegorz

Ktena, Aphrodite

Kulagin, Roman

Kulinich, Sergei

Kumar, Anil

Kumar, Avneesh

Kumar, Pankaj

Kumar, Sandeep

Kumar, Vivek

Kumavor, Patrick D.

Kung, Yu-Chun

Kuo, Wen-cheng

Kurlyandskaya, Galina V.

Kwok, Chee Yee

Kwon, Kye-Si

Kwon, Soon-Hong

Kwon, Sungkyu

La Parola, Valeria

Lacava, Cosimo

Lagarde, Florence

Lai, Chien-Chih

Lai, Chin Wei

Lai, Wen Cheng

Lai, Ying-Chih

Lam, Raymond

Lang, Udo

Lang, Walter

Lao, Ka Un

Lauga, Eric

Laurenti, Marco

Lauritano, Dorina

Lavrentovich, Oleg

Lawrie, Benjamin

Layani, Michael

Lazarus, Nathan

Le Donne, Alessia

Le Gac, Séverine

Le Pioufle, Bruno

Le Traon, Olivier

Le, NamTuan

Leccese, Fabio

Lechuga, Yolanda

Ledochowitsch, Peter

Lee, Boon Giin

Lee, Byung Chul

Lee, Changwon

Lee, Eon Soo

Lee, Hyeong Jae

Lee, Hyunjoo Jenny

Lee, Jaehyun

Lee, Jae-Ung

Lee, Jeong Hoon 
Lee, Jinkee

Lee, Jiun-haw

Lee, Keekeun

Lee, Minbaek

Lee, Seok Jae

Lee, Seung-Beck

Lee, Seung-woo

Lee, Sung Sik

Lee, Sun-Kyu

Lee, Sunwoo

Lee, Tae Yoon

Lee, Vivian

Lee, Ying-Chieh

Lei, Kin Fong

Lesch, Andreas

Levi, Alessandro

Li, Chun-Hsing

Li, Haijun

Li, He

Li, Jiashen

Li, Jinxing

Li, Lin

Li, Ming-Huang

Li, Paul C.H.

Li, Qing

Li, Sheng-Shian

Li, Tao

Li, Wei-Chang

Li, Wendi

Li, Xiang

Li, Yangmin

Li, Yifan

Li, Yiyan

Li, Yuanyuan

Li, Zhenglong

Li, Zhengwei

Li, Zida

Liang, Feng

Liang, Mengbing

Liao, Meiyong

Liao, Xiaozhou

Lim, Chun Yee

Lim, Sooman

Lin, Chih-Lang

Lin, Chih-ming

Lin, Chi-Ying

Lin, Gungun

Lin, Jr-Lung

Lin, Linhan

Lin, Yang

LIN, Yuankun

Lin, Yuan-Pin
Lin, Zuantao

Linß, Sebastian

Linzon, Yoav

Liou, Michelle

Liparoti, Sara

Lipfert, Jan

Liu, Cheng-Hsien

Liu, Han Yin

Liu, Jindong

Liu, Jun

Liu, Qingkun

Liu, Ran

Liu, Ren

Liu, Tianyu

Liu, Yang

Liu, Yazhao

Liu, Ying

Liu, Yu

Liu, Yuxin

Livermore, Carol

Lo, Yu-Hwa

López De Lacalle, Luis N.

López Villanueva, Juan Antonio

Lopez-Martinez, Maria J

Lu, Meng

Lu, Nanshu

Lu, Xinyu

Lu, Xiyuan

Lu, Yipeng

Lucarini, Gioia

Lucchetta, Daniele Eugenio

Lucibello, Andrea

Łuczak, Sergiusz

Ludwig, Kip

Luo, Jerry

Luo, Jian

Luo, Quantian

Luo, Shan

Luo, Xiaolong

Luo, Yanhua

Lutey, Adrian H.A.

Lvova, Larisa

Ma, Ji

Ma, Jiaju

Maccaferri, Nicolò

Maceiczyk, Richard

Machida, Katsuyuki

Maeda, Yusaku

Maeng, Jimin

Magdanz, Veronika

Mahshid, Sara

Makeyev, Oleksandr 
Maletinsky, Patrick

Malgaretti, Paolo

Malinauskas, Mangirdas

Malka, Dror

Mallick, Dhiman

Malm, B. Gunnar

Malviya, Kirtiman Deo

Mandal, Soumyajit

Manrique, David Zsolt

Maoz, Ben M.

Marafona, José Duarte

Marasso, Simone Luigi

Marcos, Marcos

Mariani, Stefano

Marino, Attilio

Markopoulos, Angelos P.

Markov, Dmitry

Marques, Carlos

Martel-Foley, Joseph

Martin, André

Martinez, Ramses V.

Masoero, Marco

Mastropaolo, Enrico

Materer, Nicholas F.

Matko, Vojko

Matsuhisa, Naoji

Matsukawa, Yoshitaka

Matsuo, Shigeki

Mautner, Andreas

Mavridis, Ioannis

Mazomenos, Evangelos

McHugh, Kevin

Mehdipour, Iman

Mehrabadi, Mohammad Allahdadi

Meister, Jörg

Mejia, Israel

Melo, Luís F.

Memoli, Gianluca

Meneghini, Matteo

Meng, Ellis

Meng, Fanben

Merazzo, Karla J.

Mercer, John

Meredith, Nathan

Meyer, Florent

Miah, Abdul Halim

Michael, Aron

Michalik, Jan

Miled, Amine

Mileti, Gaetano

Miluski, Piotr

Minakshi, Manickam
Miranda, Joao

Miranda, João M.

Mirea, Teona

Mirzavand, Rashid

Mishra, Aashwin

Mishra, Rupesh K.

Mistura, Giampaolo

Mitchell-Smith, Jonathon

Mitra, Srinjoy

Miura, Takashi

Mizoshiri, Mizue

Modica, Francesco

Moghimi, Mohammad

Mohamadi, Reza

Mohammad, Mahdavi

Mohd-Yasin, Faisal

Mohsen, Marani Barzani

Mondal, Kunal

Montessori, Andrea

Montgomery, Erwin B.

Montoliu Colas, Raul

Moon, Kee S.

Morales-Guio, Carlos G.

Mori, Nobuya

Morizio, James

Moschou, Despina

Mossi, Karla M.

Mouza, Aikaterini A.

$\mathrm{Mu}$, Xuan

Mulholland, Anthony

Mulla, Yusuf

Müller, Gerhard

Mulloni, Viviana

Mulvana, Helen

Munteanu, Florentina-Daniela

Muroyama, Masanori

Muszyński, Tomasz

Nabavi, Ali

Nadolny, Krzysztof

Nag, Manoj

Nagai, Moeto

Nagatani, Naoki

Nagayama, Tomonori

Naha, Pratap C.

Najarian, Siamak

Nakano, Michihiko

Nam, Yoonkey

Nama, Nitesh

Narakathu, Binu Baby

Nasiriavanaki, Mohammadreza

Nassiopoulou, Androula

Nawaz, Sarfraz 
Nazari Nejad, Saman

Nelson, Alexander H.

Neri, Giovanni

Newcomb, Robert

$\mathrm{Ng}$, Alphonsus

Nguyen, Duc Thanh

Nguyen, Thanh D.

Nguyen, Tien Dat

Nguyen, Tu (Ryan)

$\mathrm{Ni}$, Guangxin

Nian, Qiong

Nicolay, Pascal

Nieto, Daniel

Nightingale, Adrian

Niklaus, Frank

Nikolka, Mark

Nikumb, Suwas

Nilghaz, Azadeh

Nirichan, Sanoj Rejinold

Niu, Ran

Nivedita, Nivedita

Niwano, Michio

Nomura, Ken-ichi

Nordin, Gregory P.

Nose, Toshiaki

Nosrati, Reza

Novak, Richard

Nunes, Janine

O'Mahony, Conor

Obata, Kotaro

Obikawa, Toshiyuki

Ochowiak, Marek

Ogawa, Shinpei

Oh, Min-cheol

Ohlckers, Per

Ok, Jong G

Oliveira, Sara

Olson, Elizabeth S.

O'mullane, Anthony

Orsini, Andrea

Ortega-Casanova, Joaquín

Ortolani, Michele

Ostadhassan, Mehdi

Ota, Hiroki

Oxley, Thomas

Pachauri, Vivek

Padmanabhan, Jagannath

Pagliara, Stefano

Pagonis, Dimitrios - Nikolaos

Paiè, Petra

Pak, On Shun

Palchoudhury, Soubantika
Palumbo, Fabio

Pan, Zhiliang

Pandey, Santosh

Pandraud, Gregory

Pani, Danilo

Pantano, Maria

Pantoli, Leonardo

Papadakis, Christine

Park, Byung-Wook

Park, Choongbae

Park, Chun Gwon

Park, Hong-Gyu

Park, Jang-Ung

Park, Je-Kyun

Park, Jonghyun

Park, Joontaek

Park, Jung-Dong

Park, Junyong

Park, Sungsu

Park, Yong Il

Parker, Stewart F.

Passilly, Nicolas

Patel, Saurin

Patelli, Alessandro

Patterson, Jennifer

Pattini, Francesco

Pauliukaite, Rasa

Pawlak, Michał

Payam, Amir Farrokh

Pedersen, Henrik (Sweden)

Pedersen, Henrik (USA)

Peijnenburg, Willie J. G. M.

Peiner, Erwin

Pekarovicova, Alexandra

Pellet, Claude

Peng, Guiming

Peng, Zhili

Pennathur, Sumita

Pennazza, Giorgio

Pereira Neves, Hercules

Peres, António M.

Pérez-Juste, Ignacio

Perpiña, Xavier

Perrault, Cecile

Persano, Anna

Petruska, Andrew

Pettersson, Anders

Peyman, Sally A.

Pezzinga, Giuseppe

Pfattner, Raphael

Pfohl, Thomas

Pham, Duc Truong 
Pham, Phuong

Phan, Hoang-Phuong

Phan, Manh-Huong

Picollo, Federico

Piedade, Ana Paula

Pieri, Francesco

Pinho, Diana

Pinho, Luís

Pinteala, Mariana

Pinto, Artur M.

Pirozzi, Salvatore

Pitto-Barry, Anaïs

Plochberger, Birgit

Podder, Pranay

Poenar, Daniel

Poletkin, Kirill

Polimeni, Antonio

Pongrácz, Anita

Poot, Menno

Pope, Tom

Prakash, Shaurya

Prefasi, Enrique

Printz, Adam D.

Priye, Aashish

Proietti, Emanuela

Psychogiou, Dimitra

$\mathrm{Pu}$, Suan Hui

Puchades, Ivan

Pullano, Salvatore Andrea

Qamar, Afzaal

Qasaimeh, Mohammad A.

Qian, Zhenyun

Qin, Hantang

Qin, Yiheng

Qiu, Hao

Qiu, Tian

Qiu, Yinghua

Qiu, Zhen

Qu, Hongwei

Quagliata, Luca

Que, Long

Rabinovici, Raul

Radhakrishna, Ujwal

Raducanu, Bogdan C.

Raeis Hosseini, Niloufar

Raffo, Antonio

Raj, Saurabh

Rajaraman, Swaminathan

Rajnicek, Ann

Ramanavicius, Arunas

Ramini, Abdallah

Ramos, Daniel
Ranga, Adrian

Rao, Smitha

Rapp, Bastian E.

Ravasio, Chiara

Reboud, Julien

Regev, Michael

Reid, Russell C.

Reig, Càndid

Reigh, Shang Yik

Reineck, Philipp

Reinert, Wolfgang

Reinhard, Friedemann

Ren, Hao

Ren, Yong

Ren, Yukun

Renevey, Philippe

Retterer, Scott T.

Reyes-Garcés, Nathaly

Reyssat, Etienne

Rezai, Pouya

Rezk, Amgad

Rhallabi, Ahmed

Rhee, Huinam

Rhi, Seok-ho

Rho, Junsuk

Riha, Shannon C.

Rimoldi, Luca

Ristic, Michael

Rivadeneyra, Almudena

Riveros, Raul

Rizal, Conrad

Robinson, Jacob T.

Rocha, Luís A.

Rodger, Damien C.

Rodriguez, Noel

Rohac, Jan

Rokosz, Krzysztof

Romano, Lucia

Rosa, Vinicius

Rosengarten, Gary

Rosenkranz, Andreas

Rosenzweig, Derek

Rossi, Massimiliano

Rostami, Behnam

Rothbauer, Mario

Rout, Bhimsen

Rufer, Libor

Ruffato, Gianluca

Ruffino, Francesco

Rughoobur, Girish

Rurali, Riccardo

Russell, Christopher 
Rusu, Cristina

Ruther, Patrick

Rydosz, Artur

Ryu, Hyunryul

Sabater, Carlos

Sabater-Navarro, José M.

Sabhachandani, Pooja

Sadowski, Łukasz

Saeidi, Kamran

Saez, Ignacio

Šafarič, Riko

Sagués, Francesc

Saharan, Lalita

Sahoo, Deepak

Sahu, Debashish

Saito, Akira

Saito, Ken

Saito, Shinichi

Sajid, Muhammad

Sakata, Shuzo

Sakharova, Nataliya

Sakudo, Akikazu

Salek, Mohammad Mehdi

Salem, Bassem

Saltaren, Roque

Saluk-Bijak, Joanna

Samiei, Ehsan

Sankaran, Kamatchi Jothiramalingam

Santecchia, Eleonora

Santiranjan Shannigrahi, Santiranjan

Sanyal, Arindam

Sardina, Gaetano

Sardini, Emilio

Saremi, Mehdi

Sarkar, Kausik

Sato, Kimiyasu

Saxena, Tarun

Schenk, Harald

Schintke, Silvia

Schirhagl, Romana

Schmidt, Oliver G.

Schmitt, Katrin

Schmitt, Michael

Schneckenburger, Herbert

Schneider, Ben H.

Schneider, Michael

Scholten, Kee

Scholz, Ferdinand

Schulz, Sebastian Andreas

Schwaminger, Sebastian

Scott, Amy M.

Sebastian, Victor
Sedlmeir, Florian

Segreto, Tiziana

Seker, Erkin

SEKINE, Tomohito

Selyanchyn, Roman

Semaltianos, Nikolaos G.

Semprebon, Ciro

Seo, Jihoon

Seo, Jongmo

Seok, Tae Joon

Sepúlveda, Nelson

Serpooshan, Vahid

Serra, Christophe A.

Serrano-Aroca, Ángel

Serry, Mohamed

Sethian, James A.

Sethu, Palaniappan

Setti, Dinesh

Seymour, John

Shah, Pratikkumar

Shahab, Shima

Shalaby, Mohamed

Shan, Xuechuan

Shang, Luoran

Shao, Lei

Sharma, Deepti

Sharma, Swati

Shaw, Kirsty

Shayesteh Moghaddam, Narges

Shaygan, Mehrdad

Shemelya, Corey

Shen, Xiling

Sherrott, Michelle C.

Sherwood, Joseph M.

Shi, Hongliang

Shields IV, C. Wyatt

Shields, Wyatt

Shih, Steve

Shih, Wen-Pin

Shikida, Mitsuhiro

Shimada, Keita

Shimazoe, Kenji

Shimizu, Kazuo

Shin, Sangwoo

Shoffstall, Andrew

Shrirao, Anil B.

Shyu, Jin-Cherng

Sidorenko, Alexander

Sieber, Ingo

Sier, Cornelis F.M.

Siller, Hector R.

Silva, Francisco J. G. 
Simmchen, Juliane

Simpson, M. Cather

Singh, Abhalaxmi

Sirutkaitis, Valdas

Smulko, Janusz

So, Hongyun

Soejima, Tetsuro

Sohgawa, Masayuki

Sohn, Jung Woo

Soin, Navneet

Soltani, Ali

Somà, Aurelio

Son, Donghee

Son, Seung Uk

Song, Jingfeng

Song, Juha

Song, $\mathrm{Xu}$

Song, Yong-Ak (Rafael)

Song, Young Min

Songmene, Victor

Sonker, Mukul

Sorgato, Marco

Soria, Silvia

Sortino, Marco

Sosnowski, Tomasz Robert

Speghini, Adolfo

Spencer, Nicholas

Spiers, Adam

Stassi, Stefano

Stavrakis, Stavros

Stavroulakis, Georgios E.

Steenson, David Paul

Steiner, Harald

Sterken, Tom

Stiharu, Ion

Stine, Keith J.

Stolov, Andrei A.

Stratakis, Emmanuel

Stringer, Jonathan Edward

Strohm, Eric

Struk, Przemyslaw

$\mathrm{Su}$, Zhijuan

Subramani, Thiyagu

Suess, Ryan J.

Sullivan, John

Sun, Chen

Sun, Dali

Sun, Hongyu

Sun, Jian

Sun, Keye

Sun, Kien Wen

Sun, Meng
Sun, Shijing

Sun, Xiankai

Sun, Yung-Shin

Sung, Jaeyong

Sunter, Jack D.

Suzuki, Kenichiro

Svetovoy, Vitaly B.

Swami, Nathan

Syam, Wahyudin

Szczepanik, Beata

Sznitman, Josue

Szulczyński, Bartosz

Szymborski, Tomasz

Tabib-Azar, Massood

Taboryski, Rafael

Taboryski, Rafael J.

Tabrizian, Roozbeh

Tadmor, Rafael

Tahara, Hironobu

Taheri-Tehrani, Parsa

Takahashi, Hidetoshi

Takashima, Yuzuru

Talaat, Ahmed

Taler, Jan

Tallaire, Alexandre

Tamarin, Ollivier

Tan, Chee-Keong

Tan, Jifu

Tan, Lling Lling

Tan, Say

Tanabe, Katsuaki

Tanaka, Yo

Tang, Jinyao

Tang, Shiyang

Tang, Wilkin

Tanikawa, Tomoyuki

Tanyeri, Melikhan

Tao, Kai

Tao, Lei

Tardin, Catherine

Tasco, Vittorianna

Tassieri, Manlio

Tatar, Erdinc

Tavacoli, Joe W.

Temel, Fatma Zeynep

Temiz, Yuksel

Testa, Genni

Tetienne, Jean-Philippe

Thalhammer, Gregor

Thielemann, Christiane

Thijssen, Jos

Thorsen, Todd 
Tian, Yingtao

Tibbetts, Katharine Moore

Tibbitt, Mark

Tiemann, Michael

Tittonen, Ilkka

Toan, Nguyen Van

Toda, Masaya

Todorov, Tchavdar

Todorov, Yanko

Tognarelli, Selene

Tonacci, Alessandro

Torres-Diaz, Isaac

Torrilhon, Manuel

Tortschanoff, Andreas

Torunbalci, Mustafa Mert

Tosello, Guido

Toyama, Shigeru

Trotta, Gianluca

Trucco, Andrea

Trung, Tran Quang

Truong, Vi Khanh

Trusiak, Maciej

Tsai, Ang-Chen

Tsai, Chia-Hung

Tsai, Ming-Yi.

Tsamis, Christos

Tsay, Chien-Yie

Tseng, Kuo-Hsiung

Tseng, Sheng-Hsiang

Tseng, Victor Farm-Guoo

Tseng, Wei-Tsu

Tserepi, Angeliki

Tsiokos, Dimitris

Tsuchiya, Yoshishige

Tsukamoto, Takashiro

Tullis, Iain

Tung, Chih-Kuan

Twaha, Ssennoga

Tzounis, Lazaros

Uchikoba, Fumio

Ueda, Taro

Ulyashin, Alexander G.

Ulz, Peter

Um, Handon

Umasankar, Yogeswaran

Urban, Matthew W.

Uspal, William

Uzunlar, Erdal

VAI, Mang I

Valli, Angelo

Van De Put, Maarten

Van Der Meer, Th.H.
Van Der Sluis, Olaf

Van Erps, Jurgen

Van Ostayen, Ron A.J.

Van Roij, Rene

Vanfleteren, Jan

Vara, Dina

Varghese Chacko, Jenu

Vasdekis, Andreas E.

Vazquez, Maribel

Vazquez, Rebeca Martinez

Velha, Philippe

Venkataraman, Shrinivas

Verd, Jaume

Verotti, Matteo

Vezzoli, Andrea

Vidal-Álvarez, Gabriel

Vidic, Jasmina

Viefhues, Martina

Vignola, Joseph F.

Vila-Comamala, Joan

Vilela, Diana

Vinayakumar, K B

Viola, Fabio

Viollet, Stéphane

Visaveliya, Nikunjkumar

Vitry, Pauline

Volpe, Giovanni

Volz, Thomas

Vourdas, Nikolaos

Wada, Ken-Ichi

Waddell, Emanuel

Walczak, Rafał

Walling, Jeffrey

Wan, Alwin

Wang, Cheng

Wang, Dung-An

Wang, Gang

Wang, Guoan

Wang, Haidong

Wang, Hao

Wang, Hongbo

Wang, Jiangxin

Wang, Jing

Wang, Ling

Wang, Peng

Wang, Pengtao

Wang, Qi

Wang, Qiugu

Wang, Tao

Wang, Wei

Wang, Xiaoen

Wang, Xiaoguang 
Wang, Xiaomu

Wang, Ya

Wang, Yeqing

Wang, Zhenfeng

Wang, Zhisong

Ward, Tom

Ware, Taylor

Warkiani, Majid Ebrahimi

Wasserman, Daniel

Waugh, David Garreth

Wei, Kaya

Weiland, James

Weinbub, Josef

Weisensee, Patricia

Weng, Xuan

Whitby, Catherine P

Whyte, Graeme

Wieringa, Fokko

Williams, Stuart J.

Witte, Hartmut

Wlodkowic, Donald Wlodzimierz

Wojtasiak, Wojciech

Woo, Jong-Kwan

Wood, Graham S.

Wood, Troy D.

$\mathrm{Wu}$, Chengyuan

$\mathrm{Wu}$, Guoqiang

$\mathrm{Wu}$, Heng

$\mathrm{Wu}$, Hung-Chin

$\mathrm{Wu}$, Jiandong

$\mathrm{Wu}, \mathrm{Kai}$

$\mathrm{Wu}, \mathrm{Nan}$

$\mathrm{Wu}$, Tao

Wu, Xiaojian

$\mathrm{Wu}, \mathrm{Xin}$

$\mathrm{Wu}$, Zhiguang

Wuethrich, Alain

$\mathrm{X}$, Weixian

Xia, Wenjie

Xiang, Limin

Xianyu, Yunlei

Xiao, Boqi

Xie, Saien

Xie, Shangran

Xie, Yuliang

Xie, Zhijian

Xie, Zuoti

Xing, Guichuan

Xiong, Xingguo

$\mathrm{Xu}$, Jiawen

Xu, Peng

$\mathrm{Xu}$, Weihe
Xu, Weixing

$\mathrm{Xu}$, Yong

Yadav, Chandan

Yadavali, Sagar

Yagasaki, Takuma

Yahiaoui, Réda

Yamane, Daisuke

Yan, Dayun

Yan, Jing

Yan, Jiwang

Yang, Hao

Yang, Jeon-Wook

Yang, Ming

Yang, Teng-Chun

Yang, Ya-Tang

Yang, Yunze

Yano, Mitsuaki

Yao, Jeffrey

Yap, Yiing Chiing

Yapici, Murat Kaya

Yazdi, Sadegh

Ye, Enyi

Ye, Huaiyu

Yeh, Li-Hsien

Yeh, Min-Hsin

Yeh, Yin-Ting

Yen, Shih-Cheng

Yeo, Joo Chuan

Yeom, Junghoon

Yeom, Taiho

Yildirim, Adem

Yin, Xiaodong

Yoo, Byungseok

Yoon, Hyeonseok

Yoon, Hyeun Joong

Yossifon, Gilad

You, Shujie

$\mathrm{Yu}$, Chin-ping

Yu, Feiqiao Brian

$\mathrm{Yu}$, Jae Woong

$\mathrm{Yu}$, Kee-Ho

Yu, Ki Jun

$\mathrm{Yu}$, Ruei-Sung

$\mathrm{Yu}$, Xinge

$\mathrm{Yu}$, Yanhao

Yu, Yun

Yu, Zeta

Yuan, Junqi

Yuan, Lei

Yuan, Quan

Yubero, Francisco

Yue, Tao 
Yurchenko, Olena

Zaghari, Bahareh

Zagnoni, Michele

Zagórski, Ireneusz

Zahn, Jeffrey

Zaman, Ashraf Uz

Zangari, Giovanni

Zareinia, Kourosh

Zarifi, Mohammad H.

Zayachuk, Yevhen

Zeidler, Henning

Zenati, Marco A.

Zergioti, Ioanna

Zhang, Chao

Zhang, Di

Zhang, Hongti

Zhang, Huairuo

Zhang, Huanan

Zhang, Jun

Zhang, Nan

Zhang, Renyun

Zhang, Rui

Zhang, Rujing

Zhang, Wenxu

Zhang, Xian

Zhang, Xiang

Zhang, Xiao

Zhang, Xiaoliang

Zhang, Xinquan

Zhang, Xueqiang Alex

Zhang, Xuming

Zhang, Xuping
Zhang, Yanzhen

Zhang, Yi

Zhang, Yong

Zhang, Yuhao

Zhang, Yunyan

Zhang, Zhien

Zhang, Ziyang

Zhao, Chun

Zhao, Hangbo

Zhao, Hong

Zhao, Ping

Zhao, Ruogang

Zhao, Yuda

Zhao, Yulong

Zheng, Bo

Zheng, Yi

Zheng, Yuebing

Zhong, Li

Zhou, Bingpu

Zhou, Ran

Zhu, Haoshen

$\mathrm{Zhu}, \mathrm{He}$

Zhu, Lianhua

Zhu, Wei

Zhu, Ying

Zimmerman, William B.

Zolfagharian, Ali

Zotti, Linda

Zozulya, Alexey

Zuo, Lei

Zuo, Lijian

Župerl, Uroš

(C) 2019 by the author. Licensee MDPI, Basel, Switzerland. This article is an open access article distributed under the terms and conditions of the Creative Commons Attribution (CC BY) license (http://creativecommons.org/licenses/by/4.0/). 